\title{
Emergence de la fièvre catarrhale ovine dans le Bassin méditerranéen et surveillance entomologique en France
}

\author{
T. Baldet ${ }^{1}$ B. Mathieu ${ }^{2}$ J.C. Delécolle ${ }^{3}$ \\ G. Gerbier ${ }^{1}$ F. Roger ${ }^{1}$
}

Mots-clés

Ovin - Culicoides imicola Fièvre catarrhale ovine Surveillance - Corse - France Région méditerranéenne.

\begin{abstract}
Résumé
La fièvre catarrhale ovine (FCO) est une maladie virale qui est transmise par des moucherons piqueurs (Diptera : Ceratopogonidae) et émerge en Méditerranée depuis 1998. Cette émergence est reliée à l'expansion septentrionale de son vecteur principal Culicoides imicola Kieffer 1913. En France, C. imicola a été décelé dans le sud de la Corse en octobre 2000, quelques jours avant I'apparition de foyers de FCO sérotype 2 . Un réseau de surveillance entomologique a été mis en place en 2002 : (i) en Corse, pour étudier les dynamiques de populations de $C$. imicola et des autres espèces de Culicoides associées aux élevages; (ii) sur le continent, pour détecter I'introduction de C. imicola. Des captures nocturnes ont été réalisées toutes les trois semaines sur 12 sites en Corse et une fois par mois sur 19 sites sur le continent. En 2002, en Corse, un total de 180 nuits de captures ont été effectuées. C. imicola comptait en moyenne pour 18 p. 100 des effectifs totaux capturés et prédominait au sud de I'île. Les densités adultes ont atteint un pic en septembre. La présence de C. imicola sur tous les sites et durant huit mois de l'année a confirmé que le vecteur principal de la FCO passait l'hiver et était installé durablement dans I'île. En outre, en 2003, les populations de C. imicola étaient en pleine expansion dans le Nord, particulièrement en Balagne. Le risque d'invasion du continent devient ainsi plus important. Sur le continent, sur un total de 233 nuits de captures réalisées en 2002-2003, deux spécimens de C. imicola ont été détectés en 2003 sur deux sites différents. Des investigations poussées n'ont pu mettre en évidence des populations locales installées. Les dynamiques saisonnière et spatiale de $C$. imicola et des autres espèces d'intérêt sont discutées en relation avec leur bioécologie et les facteurs environnementaux. Cette " entomosurveillance » apparaît indispensable pour une meilleure compréhension de l'épidémiologie de la FCO mais aussi pour élaborer et valider des modèles de prédiction de zones à risque.
\end{abstract}

\section{INTRODUCTION}

La fièvre catarrhale ovine (FCO), bluetongue en anglais, est une maladie virale affectant les ruminants et notamment les ovins. Son issue souvent fatale chez ces derniers en fait une maladie très grave sur le plan économique, classée au rang A de l'Organisation mondiale de la santé animale (OIE). L'agent causal est un virus de la famille des Reoviridae (genre Orbivirus), dont on connaît 24

\footnotetext{
1. Cirad, département Emvt, TA 30 E, campus international de Baillarguet, 34398 Montpellier, France

Tél. : +33 (0)4675938 68 ; fax : +33(0)4 67593754

E-mail : thierry.baldet@cirad.fr

2. EID-Méditerranée, 165 avenue Paul Rimbaud, 34184 Montpellier, France

3. Université Louis Pasteur, 29 boulevard de la Victoire, 67000 Strasbourg, France
}

sérotypes différents, ayant une répartition géographique propre, une pathogénicité variable et ne conférant pas de protection croisée. La FCO est transmise par la piqûre de moucherons femelles appartenant à certaines espèces du genre Culicoides (Diptera : Ceratopogonidae). L'aire de répartition de la FCO, dépendant étroitement de la distribution et de l'abondance de ses vecteurs, couvre tous les continents entre $40^{\circ}$ de latitude Nord et $35^{\circ}$ de latitude Sud (15). Les principales espèces vectrices sont $C$. imicola et $C$. bolitinos en Afrique, $C$. imicola et $C$. fulvus en Asie, $C$. brevitarsis et $C$. fulvus en Australie, $C$. sonorensis en Amérique du Nord, et $C$. insignis et C. pusillus en Amérique centrale et en Amérique du Sud (17). Dans les régions tropicales (aire enzootique), les races locales ovines sont naturellement peu sensibles, les manifestations cliniques sont surtout visibles sur les races ovines améliorées importées. Dans les régions subtropicales et tempérées, la FCO se manifeste sous forme de flambées épizootiques plus ou moins régulières. 
En Europe, la FCO a fait des incursions temporaires dans le passé : en Espagne et au Portugal de 1957 à 1960 (sérotype 10), à Chypre en 1977 et en Grèce (Lesbos) en 1980 (sérotype 4). Toutefois, depuis 1998 une émergence majeure concerne l'ensemble du Bassin méditerranéen $(1,18)$, avec deux voies principales d'invasion : à l'est (Grèce, Balkans), les sérotypes 1, 4, 9 et 16, avec comme origine probable la Turquie et le Proche-Orient ; à l'ouest (Sardaigne, Corse, Baléares, nord de la péninsule italienne), le sérotype 2, avec comme origine probable le Maghreb. La Sicile et le sud de la péninsule italienne, situés à l'interface de ces deux voies, doivent faire face à l'ensemble de ces sérotypes. La propagation de la maladie est liée à l'expansion septentrionale de son vecteur afro-asiatique : $C$. imicola (figure 1). Outre le fait que l'installation récente de $C$. imicola est objectivée dans de nombreuses régions du nord du Bassin méditerranéen, des travaux phylogénétiques récents (10) ont montré l'existence de deux populations de $C$. imicola distinctes : l'une dans l'ouest du bassin, l'autre dans l'est, corroborant par là même l'existence des deux voies d'invasion virale.

La bioécologie de $C$. imicola dépend étroitement des conditions climatiques (température, humidité). Les gîtes larvaires sont probablement constitués par de la matière organique en décomposition (excréments d'animaux) mais restent à caractériser. Le développement larvaire dure d'une à plusieurs semaines, selon les conditions du milieu. Des phénomènes de diapauses hivernales ou d'estivohibernation ont été décrits chez certaines espèces mais restent à étudier chez $C$. imicola. Seule la femelle adulte se nourrit de sang. Sa longévité est estimée à 10-20 jours avec un maximum de 50, naturellement conditionné par les conditions abiotiques. Son activité est essentiellement nocturne et dépend étroitement de la température, avec une plage optimale entre 18 et $38^{\circ} \mathrm{C}$ (24). La dispersion active est faible, de l'ordre de quelques centaines de mètres (17). La dispersion passive peut être très importante et aller jusqu'à 700 km selon certains auteurs $(4,16)$. Les vents incriminés sont en altitude, jusqu'à $2000 \mathrm{~m}$ et ils ont des vitesses de 10 à $40 \mathrm{~km} / \mathrm{h}$ à des températures compatibles avec la survie des insectes. En Corse, $C$. imicola a été détecté pour la première fois en octobre 2000 (12) et des foyers cliniques du virus de la bluetongue sérotype 2 (BTV-2) sont apparus en automne 2000 (49 foyers) et en été-automne 2001 (335 foyers) $(29,30,31)$. La lutte antivectorielle et les mesures de protection (application pour on et bains d'insecticide ou de répulsifs, stabulation) ne sont pas suffisantes. Seules les campagnes de vaccination et les contraintes de police sanitaire permettent de contrôler la maladie. Aussi, suite aux campagnes vaccinales conduites au

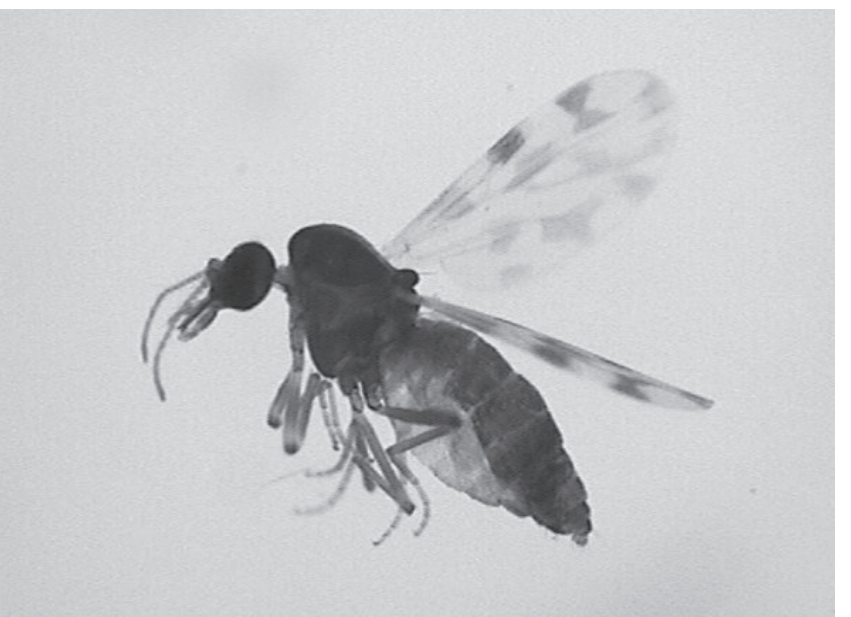

Figure 1 : Culicoides imicola, vecteur afro-asiatique de la fièvre catarrhale ovine. () J. C. Delécolle, univ. Louis Pasteur cours des hivers 2001 et 2002, aucun foyer de FCO n'a été mis en évidence en Corse en 2002. Parallèlement à ces campagnes vaccinales, un réseau de surveillance pluridisciplinaire (clinique, sérologique, virologique, entomologique) impliquant différents partenaires (Ddsv ${ }^{1}$, Afssa ${ }^{2}$, ULP ${ }^{3}$, EID-Méditerranée ${ }^{4}$, Cirad, département Emvt ${ }^{5}$ ) a été mis en place par le ministère de l'Agriculture. La surveillance entomologique vise (i) en Corse à étudier les dynamiques de population de $C$. imicola et des autres espèces de Culicoides liées aux élevages, et (ii) sur le continent à surveiller l'introduction de $C$. imicola dans les départements français riverains de la Méditerranée. Les résultats de cette «entomosurveillance » pour 2002 et 2003 sont présentés ici.

\section{MATERIEL ET METHODES}

\section{Sites d'étude}

Ont été retenues : en Corse, 12 bergeries représentatives, réparties sur l'île et affectées par le BTV-2 en 2000/2001 ; et sur le littoral continental, 19 bergeries, réparties à intervalles de $50 \mathrm{~km}$ (figure 2).

\section{Protocole de capture}

Une nuit de captures par site a été réalisée toutes les trois semaines en Corse et une fois par mois sur le continent. Pour cela, des pièges lumineux à ultraviolets $4 \mathrm{~W}, 12 \mathrm{~V}$, type Miniature New Jersey Light Trap modifié (21) ont été utilisés (figure 3 ). Le piège était placé à l'extérieur à proximité immédiate de la bergerie, accroché à $2 \mathrm{~m}$ du sol, à l'abri du vent et éloigné de toute autre source lumineuses. Il était mis en place avant le coucher du soleil et relevé au petit matin. Un flacon rempli de $300 \mathrm{ml}$ d'eau savonneuse a été adapté sous le piège. Le flacon était ramené au laboratoire où tous les insectes piégés étaient transvasés et conservés jusqu'au tri, à $4^{\circ} \mathrm{C}$, dans de l'alcool à $95^{\circ}$.

\section{Identification des Culicoides}

L'identification morphologique des différentes espèces de Culicoides piégées a été basée sur l'examen à la loupe binoculaire, à un faible grossissement, des dessins alaires. En vue d'une diagnose microscopique plus précise, certains spécimens ont été disséqués et montés entre lame et lamelle dans un milieu de montage après un traitement préalable pendant $24 \mathrm{~h}$ dans une solution saturée d'alcool à $95^{\circ}$ et de phénol, selon la technique de Wirth et Marston (27). Les clés de diagnoses élaborées par Delécolle (11) ont été utilisées. Outre les dessins alaires, les principaux caractères morphologiques employés chez les femelles sont : l'espace interoculaire, le nombre et l'arrangement des différentes sensilles des antennes, la forme du troisième segment du palpe maxillaire et de son organe sensoriel, ainsi que le nombre et la forme des spermathèques. Chez les mâles, les caractéristiques des antennes sont aussi utilisées, mais c'est surtout la forme des différentes parties de la pince génitale qui permettent la diagnose d'espèce.

Ont été ensuite dénombrés pour chaque espèce le nombre total d'individus, et le nombre de mâles et de femelles. Un site intranet gère les données de cette veille entomologique de manière à ce que tous les partenaires aient accès le plus rapidement possible à l'ensemble des données de la surveillance.

\footnotetext{
${ }^{1}$ Direction départementale des services vétérinaires

${ }^{2}$ Agence française de sécurité sanitaire des aliments

${ }^{3}$ Université Louis Pasteur de Strasbourg

${ }^{4}$ Entente interdépartementale pour la démoustication du littoral méditerranéen

${ }^{5}$ Centre de coopération internationale en recherche agronomique pour le développement, département d'Elevage et de médecine vétérinaire
} 


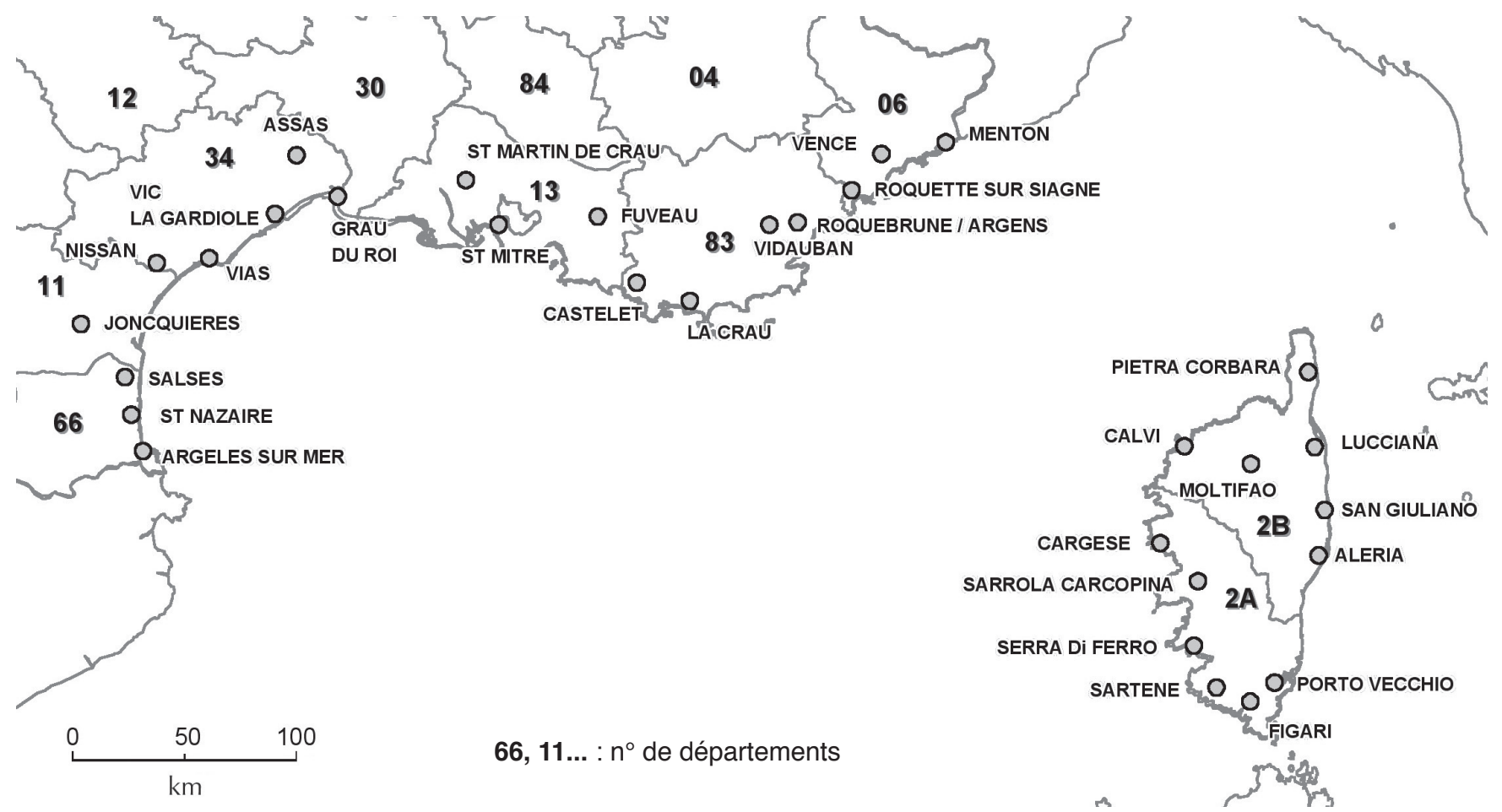

Figure 2 : localisation des sites sentinelles; surveillance entomologique de la fièvre catarrhale ovine en France (2002-2003). () T. Baldet, Cirad, dépt Emvt

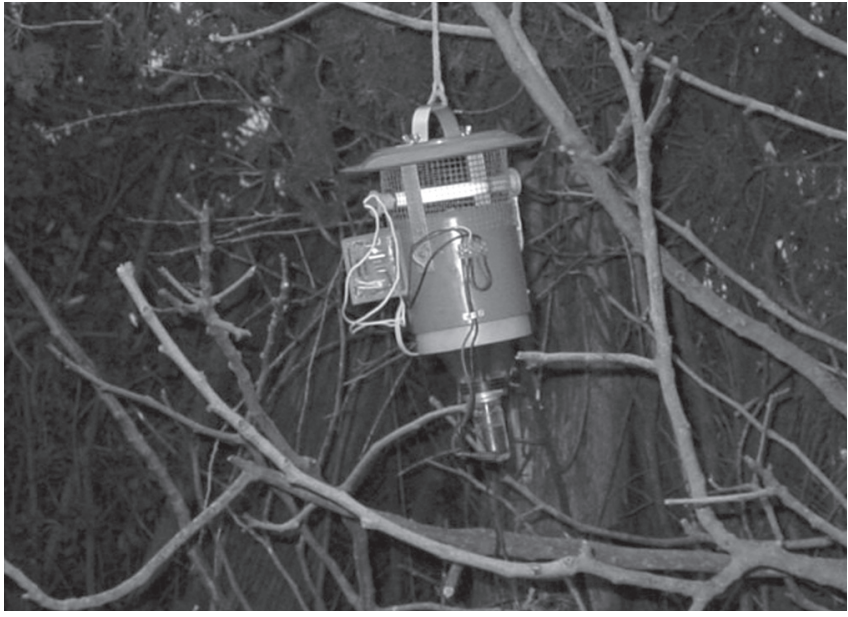

Figure 3 : piège à ultraviolets standard utilisé pour échantillonner les Culicoides.

\section{RESULTATS}

Ces résultats sont en libre accès sur le site http://blue-tongue.cirad.fr/ Index.html.

\section{Corse}

En Corse, de février à décembre 2002, 84790 Culicoides appartenant à 49 espèces différentes ont été capturés au cours de 180 nuits de piégeages (tableau I). C. imicola a représenté 18,3 p. 100 des spécimens capturés avec une moyenne de 86 moucherons par piège par nuit $(\mathrm{m} / \mathrm{p} / \mathrm{n})$. Le vecteur principal a été prédominant dans deux sites localisés à l'extrême Sud - Porto-Vecchio (moyenne de $578 \mathrm{~m} / \mathrm{p} / \mathrm{n})$ et Figari $(326 \mathrm{~m} / \mathrm{p} / \mathrm{n})$ - et le moins représenté à Moltifao
$(1 \mathrm{~m} / \mathrm{p} / \mathrm{n})$, seul site situé à l'intérieur de l'île à une altitude de $250 \mathrm{~m}$ (figure 4). Les densités adultes de $C$. imicola ont atteint un maximum en fin d'été et début d'automne (figure 5).

La présence de $C$. imicola dans tous les sites et durant huit mois de l'année (mai à décembre) a confirmé que le vecteur principal de la FCO passait l'hiver et était installé durablement dans l'île avec plusieurs générations d'adultes se succédant au cours de la saison. Les autres espèces apparues en nombre dans les piégeages ont été : $C$. newsteadi (36,8 p. 100 du total piégé, moyenne de $174 \mathrm{~m} / \mathrm{p} / \mathrm{n}), C$. scoticus $(18$ p. $100,85 \mathrm{~m} / \mathrm{p} / \mathrm{n})$, C. obsoletus $(9,2$ p. $100,43 \mathrm{~m} / \mathrm{p} / \mathrm{n})$, C. circumscriptus $(4,2$ p. $100,20 \mathrm{~m} / \mathrm{p} / \mathrm{n})$ et $C$. pulicaris $(3,8$ p. 100 , $18 \mathrm{~m} / \mathrm{p} / \mathrm{n})$. Les 43 autres espèces piégées ont compté chacune pour moins de 2 p. 100 du total piégé (tableau I).

C. newsteadi, espèce halophile, a été prépondérant dans les sites localisés en plaine lagunaire côtière (sud et ouest) et moins présent sur la côte rocheuse escarpée est et nord de l'île. $C$. pulicaris, espèce de basse à moyenne altitude, a dominé sur ladite côte escarpée ainsi qu'à l'intérieur. Les auteurs ont noté pour les espèces les plus représentées, notamment $C$. obsoletus et $C$. pulicaris, une allure bimodale des dynamiques saisonnières, avec des pics de densité adulte plus ou moins marqués : un au printemps et un en automne. Les conditions climatiques de l'été (température élevée, hygrométrie faible) pourraient être défavorables au développement larvaire et/ou au vol actif des adultes de ces espèces européennes.

En 2003, les principaux résultats ont confirmé ces tendances. En outre, les densités adultes de $C$. imicola étaient en pleine expansion dans le nord de l'île, surtout à Calvi (Balagne). Ainsi, sur les 15 nuits de piégeages effectuées en 2002 sur ce site, la moyenne en Culicoides sp. a été de $82 \mathrm{~m} / \mathrm{p} / \mathrm{n}$ dont 16 pour C. imicola (20 p. 100 du total). En 2003, sur les 16 nuits de piégeages réalisées sur le même site, la moyenne en Culicoides sp. a été de $204 \mathrm{~m} / \mathrm{p} / \mathrm{n}$ dont 153 pour $C$. imicola (75 p. 100 du total). Entre les deux années, la moyenne pour les espèces européennes (toutes sauf C. imicola $)$ piégées à Calvi a été très comparable $(66 \mathrm{~m} / \mathrm{p} / \mathrm{n}$ en 2002 


\section{Tableau I}

Résultats des piégeages réalisés en Corse et sur le continent (2002-2003)

\begin{tabular}{|c|c|c|c|c|c|c|c|c|}
\hline \multirow{3}{*}{$\begin{array}{l}\text { Année } \\
\text { Espèce }\end{array}$} & \multicolumn{4}{|c|}{ Corse } & \multicolumn{4}{|c|}{ Continent } \\
\hline & \multicolumn{2}{|c|}{2002} & \multicolumn{2}{|c|}{2003} & \multicolumn{2}{|c|}{2002} & \multicolumn{2}{|c|}{2003} \\
\hline & $\begin{array}{c}\text { Effectifs } \\
\text { piégés } \\
{[\% \text { du total }]}\end{array}$ & $\begin{array}{l}\text { Moyenne } \\
\text { de } \\
\text { piégeages a }\end{array}$ & $\begin{array}{c}\text { Effectifs } \\
\text { piégés } \\
\text { [\% du total] }\end{array}$ & $\begin{array}{c}\text { Moyenne } \\
\text { de } \\
\text { piégeages }^{\text {a }}\end{array}$ & $\begin{array}{c}\text { Effectifs } \\
\text { piégés } \\
\text { [\% du total] }\end{array}$ & $\begin{array}{c}\text { Moyenne } \\
\text { de } \\
\text { piégeages }^{a}\end{array}$ & $\begin{array}{c}\text { Effectifs } \\
\text { piégés } \\
\text { [\% du total] }\end{array}$ & $\begin{array}{c}\text { Moyenne } \\
\text { de } \\
\text { piégeages }^{\mathrm{a}}\end{array}$ \\
\hline C. imicola & $15530[18,3]$ & 86,3 & $63642[57,9]$ & 365,8 & $0[-]$ & - & $2[0,02]$ & 0,02 \\
\hline C. newsteadi & $31224[36,8]$ & 173,5 & $24458[22,3]$ & 140,6 & $11898[73,5]$ & 109,2 & $3838[44,3]$ & 31,0 \\
\hline C. obsoletus & $7762[9,2]$ & 43,1 & $1332[1,2]$ & 7,7 & $1290[8,0]$ & 11,8 & $1421[16,4]$ & 11,5 \\
\hline C. scoticus & $15280[18,0]$ & 84,9 & $7239[6,6]$ & 41,6 & $877[5,4]$ & 8,0 & $966[11,1]$ & 7,8 \\
\hline C. pulicaris & $3229[3,8]$ & 17,9 & $4097[3,7]$ & 23,5 & $188[1,2]$ & 1,7 & $113[1,3]$ & 0,9 \\
\hline C. circumscriptus & $3571[4,2]$ & 19,8 & $1861[1,7]$ & 10,7 & $526[3,2]$ & 4,8 & $550[6,3]$ & 4,4 \\
\hline C. griseidorsum & $1558[1,8]$ & 8,7 & $804[0,7]$ & 4,6 & $430[2,7]$ & 3,9 & $369[4,3]$ & 3,0 \\
\hline C. subfagineus & $1141[1,3]$ & 6,3 & $932[0,8]$ & 5,4 & $173[1,1]$ & 1,6 & $61[0,7]$ & 0,5 \\
\hline C. lupicaris & $1140[1,3]$ & 6,3 & $1154[1,0]$ & 6,6 & $178[1,1]$ & 1,6 & $1[0,01]$ & 0,01 \\
\hline Culicoides sp. & $4355[5,1]^{b}$ & 24,2 & $4416[4,0]^{\mathrm{c}}$ & 25,4 & $637[3,96]^{f}$ & 5,8 & $1347[15,5]^{\mathrm{g}}$ & 10,8 \\
\hline Total Culicoides & $84790[100]^{d}$ & 471,1 & $109935[100] \mathrm{e}$ & 631,8 & $16197[100]^{\mathrm{h}}$ & 148,6 & $8666[100]^{i}$ & 69,9 \\
\hline
\end{tabular}

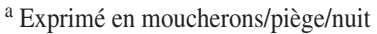
Corse

2002 : 180 nuits de piégeages (12 sites, 11 mois)

$2003: 174$ nuits de piégeages ( 12 sites, 12 mois)

b 40 autres espèces comptant chacune pour moins de $1 \%$ du total piégé

c 45 autres espèces comptant chacune pour moins de $1 \%$ du total piégé

d Total de 49 espèces de Culicoides piégés

e Total de 54 espèces de Culicoides piégés

\section{Continent}

2002: 109 nuits de piégeages (19 sites, 8 mois)

$2003: 124$ nuits de piégeages ( 19 sites, 8 mois)

f 36 autres espèces comptant chacune pour moins de $1 \%$ du total piégé

g 26 autres espèces comptant chacune pour moins de $1 \%$ du total piégé

h Total de 44 espèces de Culicoides piégés

i Total de 35 espèces de Culicoides piégés

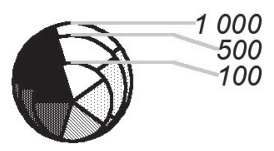

C. imicola

C. newsteadi

$\square$ C. obsoletus

C. pulicaris

Culicoides autres
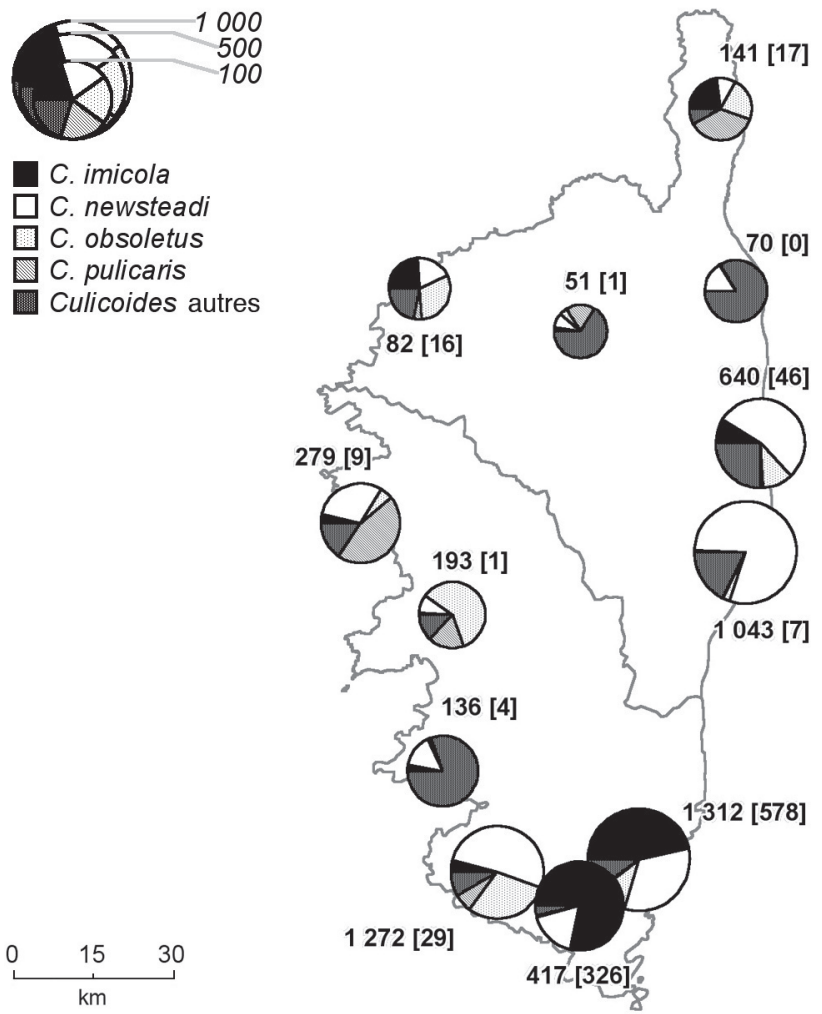

Figure 4 : distribution spatiale des Culicoides piégés en Corse de février à décembre 2002 (12 sites / 11 mois / 180 nuits de piégeages) ; 417 [326] : moyenne de piégeages en moucherons/piège/nuit (dont $\mathrm{C}$. imicola). et $51 \mathrm{~m} / \mathrm{p} / \mathrm{n}$ en 2003). Le rendement du piège n'était pas à mettre en cause, les populations locales de $C$. imicola ont été multipliées par 10 en une seule année.

\section{Continent}

Sur le continent, d'avril à novembre 2002, 16197 Culicoides appartenant à 44 espèces différentes ont été capturés au cours de 109 nuits de piégeages (tableau I). Aucun spécimen de $C$. imicola n'a été récolté. Cette absence du vecteur principal de la FCO a été corroborée par l'absence de signes cliniques et/ou de sérologies positives pour la FCO. Les espèces qui sont apparues en nombre dans les piégeages ont été : $C$. newsteadi $(73,5$ p. 100 du total piégé, moyenne de $109 \mathrm{~m} / \mathrm{p} / \mathrm{n})$, C. obsoletus (8 p. 100, $12 \mathrm{~m} / \mathrm{p} / \mathrm{n})$, C. scoticus $(5,4$ p. $100,8 \mathrm{~m} / \mathrm{p} / \mathrm{n})$, C. circumscriptus $(3,2$ p. $100,5 \mathrm{~m} / \mathrm{p} / \mathrm{n})$ et $C$. griseidorsum (2,7 p. 100, $4 \mathrm{~m} / \mathrm{p} / \mathrm{n})$. Les 39 autres espèces ont compté chacune pour moins de 2 p. 100 du total (tableau I).

Le classement a été comparable à celui observé en Corse avec toutefois pour chaque espèce des densités adultes moyennes moindres. A l'instar de la Corse, $C$. newsteadi a été prépondérant dans les plaines littorales lagunaires de l'ouest du littoral méditerranéen Pyrénées-Orientales (département $\left.n^{\circ} 64\right)$, Aude $\left(n^{\circ} 11\right)$, Hérault $\left(n^{\circ} 34\right)$ et Bouches-du-Rhône $\left(n^{\circ} 13\right)$ - et $C$. pulicaris relativement plus abondant dans les contreforts des Pyrénées et des Alpes (figure 6). Pour $C$. obsoletus et $C$. pulicaris, a été relevée comme en Corse une allure bimodale des dynamiques saisonnières avec deux pics, l'un au printemps et l'autre en automne (figure 7). En 2003, les auteurs ont pu mettre en évidence pour la première fois deux spécimens de $C$. imicola sur le littoral méditerranéen français : un spécimen mâle capturé dans la nuit du 22 mai 2003 sur le site du 
Castellet (43 12' 16" lat. N, 5 45' 31" long. E, altitude $199 \mathrm{~m}$ ) et un spécimen femelle capturé dans la nuit du 24 septembre 2003 sur le site de La Roquette-sur-Siagne ( $43^{\circ} 34^{\prime}$ ' $38^{\prime \prime}$ lat. N, $6^{\circ} 56^{\prime}$ 07" long. E, altitude $10 \mathrm{~m}$ ). Dans les deux cas, des recherches entomologiques poussées réalisées autour de ces deux sites n'ont
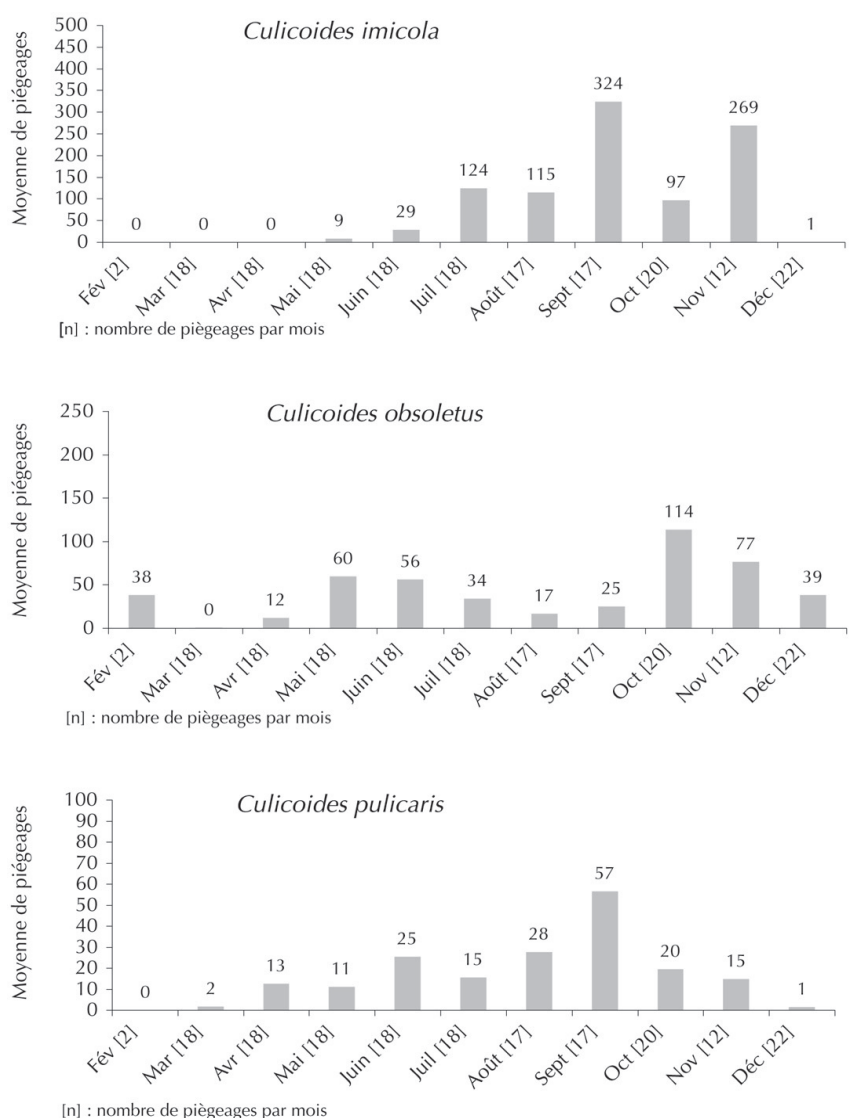

Figure 5 : dynamique saisonnière de $\mathrm{C}$. imicola, C. obsoletus et C. pulicaris en Corse en 2002. Nota : l'échelle des ordonnées (moyenne de piégeages en moucherons/piège/nuit) est différente selon les espèces. pas permis de déceler d'autres spécimens. Le suivi entomologique sentinelle sur les autres sites et sur ces deux sites les mois suivants n'a pas permis d'isoler d'autres spécimens de $C$. imicola. Dans le même temps, des contrôles sérologiques réalisés sur les espèces sensibles au niveau de ces deux sites se sont tous révélés négatifs.

\section{DISCUSSION}

En 2002, le gradient sud-nord des populations de C. imicola observé en Corse s'expliquait probablement par la colonisation récente à partir de la Sardaigne. D'une manière plus générale, les différences de répartition spatiale relevées pour $C$. imicola mais aussi pour les autres espèces d'intérêt (C. obsoletus, $C$. pulicaris) reposaient sur (i) la typologie des sites de piégeages retenus et (ii) les conditions environnementales (climat, pédologie...) liées à chacune de ces zones géographiques et favorables à l'une ou l'autre de ces espèces. Aussi, $C$. imicola est décrit en Méditerranée comme une espèce essentiellement littorale inféodée aux élevages $(5,7$, $19,22,25)$ avec une limite de répartition en altitude de l'ordre de $800 \mathrm{~m}(20)$.

L'expansion septentrionale de $C$. imicola est un phénomène dynamique en cours actuellement, comme en témoigne l'accroissement de ses populations en Balagne. Cette région enclavée du nord-ouest de la Corse est située à moins de $200 \mathrm{~km}$ des côtes varoises. Le risque d'invasion du littoral continental français est par conséquent accru. La découverte originale de deux spécimens de $C$. imicola, l'un dans le Var et l'autre dans les Alpes-Maritimes en 2003, sans évidence pour le moment de populations installées, corrobore cette suspicion. A priori, les quelques spécimens adultes introduits n'ont pas trouvé les conditions environnementales favorables à l'établissement d'une population locale. La notion d'effet fondateur - population minimale nécessaire à une implantation pérenne dans un contexte particulier - est vraisemblablement à prendre en compte. Dans les pays voisins de la France, les éléments ci-après ont été constatés en 2003 concernant la colonisation de nouveaux territoires par $C$. imicola. Dans le nord de la péninsule italienne (Ligurie, région de Gênes), depuis le démarrage de la surveillance entomologique en 2000, seuls six spécimens de C. imicola ont été récoltés (Accorsi et Mignonne, commun. pers.). Il s'agit probablement, comme pour

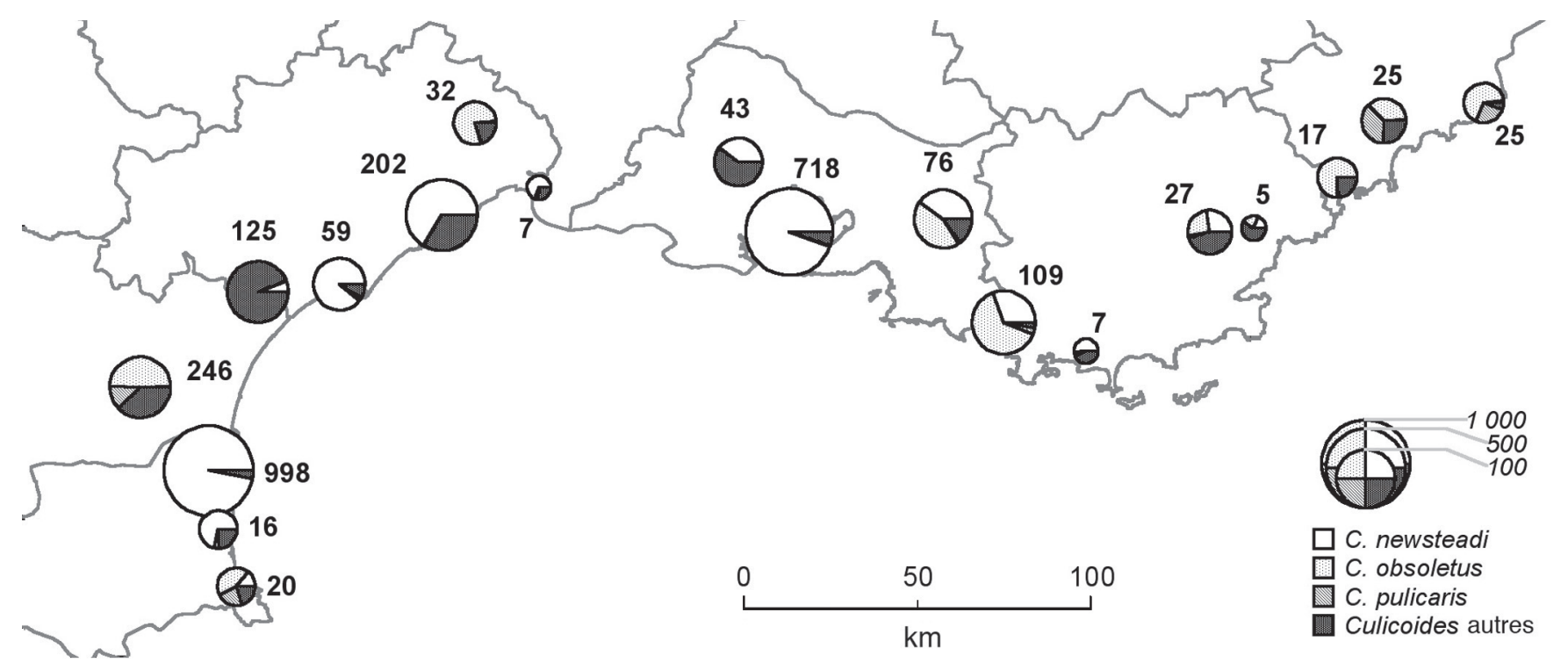

Figure 6 : distribution spatiale des Culicoides piégés sur le continent d'avril à novembre 2002 (19 sites / 8 mois / 109 nuits de piégeages) ; 998 : moyenne de piégeages en moucherons/piège/nuit ; aucun spécimen de C. imicola piégé. 

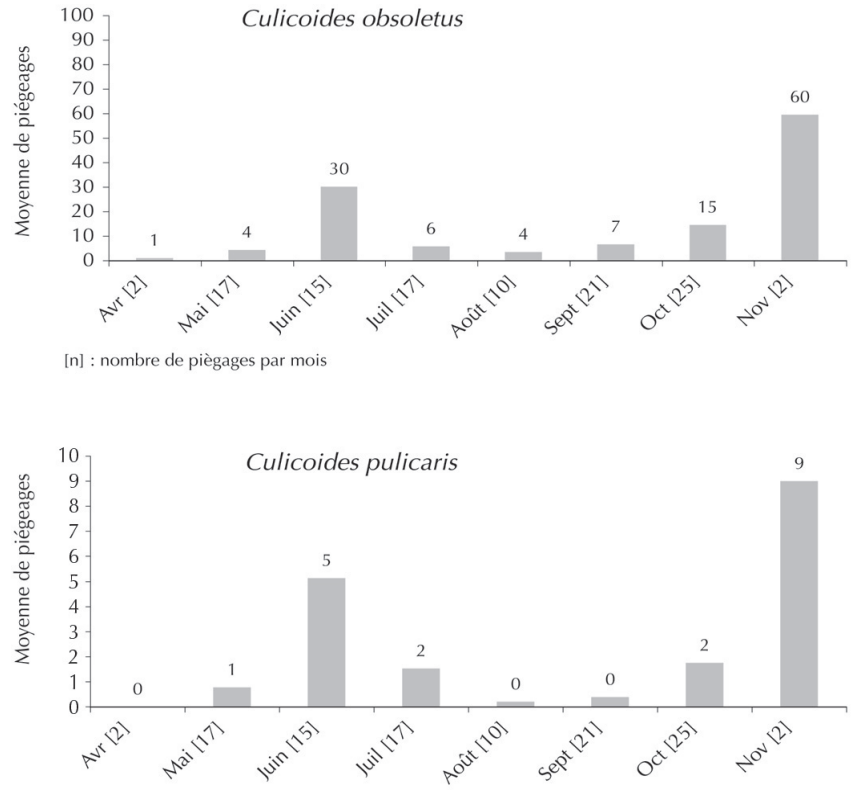

[n] : nombre de piègages par mois

Figure 7 : dynamique saisonnière de $\mathrm{C}$. obsoletus et $\mathrm{C}$. pulicaris sur le continent en 2002. Nota : l'échelle des ordonnées (moyenne de piégeages en moucherons/piège/nuit) est différente selon les espèces.

la France continentale, d'individus erratiques en provenance soit de régions plus méridionales (Toscane, Latium) où $C$. imicola est déjà installé, soit directement de Corse. Ceci relativise provisoirement le risque d'invasion de la France par la frontière italienne. A l'inverse, C. imicola a envahi au cours de l'été 2003 la Catalogne espagnole alors qu'un seul spécimen avait été capturé en 2002 dans la même région (Sarto I Monteys, commun. pers.). Des contacts ont été établis avec les responsables de cette surveillance pour objectiver cette colonisation très récente issue probablement des îles Baléares, afin d'évaluer le risque éventuel pour la région frontalière des PyrénéesOrientales. La découverte d'un spécimen de $C$. imicola a également été notée en Suisse à une latitude de $44^{\circ} 30^{\prime} \mathrm{N}$ (maximum septentrional jamais recensé) dans une région de faible altitude frontalière du nord de l'Italie (Cagienard, commun. pers.).

Les vents du sud seraient à l'origine de la remontée de $C$. imicola en Méditerranée, cet insecte pouvant être diffusé passivement comme du "plancton aérien » sur de longues distances à l'instar d'autres espèces pour lesquelles de tels phénomènes ont été notés, comme Simulium damnosum sl, vecteur de l'onchocercose ou cécité des rivières en Afrique de l'Ouest (13). La pérennisation de populations locales survient à la faveur de conditions environnementales favorables. Probablement des spécimens de $C$. imicola ont pu par le passé pénétrer dans les îles méditerranéennes à partir du Maghreb, où l'espèce est signalée depuis de nombreuses années, notamment au Maroc sous le nom de C. pallidipennis (2). En Corse, une première note sur la faune culicoïdienne (14) signale la présence de 11 espèces. Cette étude princeps, basée sur des piégeages de jour et des émergences, obtenues à partir de prélèvements de biotopes larvaires rapportés au laboratoire, n'avait pas mis en évidence la présence de $C$. imicola. L'installation de $C$. imicola en Corse est donc probablement récente, la mise en évidence de populations locales étant survenue à la faveur de l'introduction du sérotype 2 et de foyers cliniques en Sardaigne, puis en Corse au cours de l'automne 2000 (12). C. sahariensis (= C. coluzzii), autre espèce décrite en Algérie, signalée par erreur dans les années 1960 sous le nom de $C$. similis au Maroc, en Tunisie et en Italie (6), a été trouvé récemment sur le continent au cours de cette surveillance alors qu'il n'avait jamais été recensé par le passé.

Le rôle du réchauffement climatique dans ces colonisations récentes reste à objectiver. Des projections ont évalué que la température moyenne augmenterait de l'ordre de $2{ }^{\circ} \mathrm{C}$ d'ici l'année 2100 , avec une incertitude comprise entre 1,5 et $6{ }^{\circ} \mathrm{C}$ (26). Ce réchauffement global équivaudrait dans les régions tempérées à un déplacement des isothermes actuelles de 150 à $550 \mathrm{~km}$ en latitude et de 150 à $550 \mathrm{~m}$ en altitude. Des modèles récents basés sur une augmentation de la température de $2{ }^{\circ} \mathrm{C}$ ont montré qu'une grande partie de l'Europe méridionale, et notamment la France continentale, était susceptible à l'avenir d'héberger des populations locales de $C$. imicola (28). Outre son expansion spatiale et saisonnière, une augmentation de la température combinée à des modifications du régime des précipitations favoriseraient également la capacité vectorielle de C. imicola, avec notamment (i) une diminution de la durée de développement des stades larvaires, (ii) une diminution de la durée de l'amplification virale au sein du vecteur, (iii) une augmentation du nombre de repas sanguins et du nombre de pontes, et (iv) un accroissement de la durée de vie du vecteur.

En zone tempérée, les densités de $C$. imicola sont maximales en fin d'été, donnant par là même une saisonnalité marquée à la maladie. Le tassement observé en octobre 2002 en Corse pourrait être lié aux précipitations orageuses que connaissent les climats méditerranéens en septembre. Ces orages entraîneraient un lessivage des gîtes larvaires et une diminution conséquente des émergences se reflétant avec un décalage temporel sur les densités adultes. La baisse brutale des températures survenant alors pourrait aussi ralentir le développement larvaire et l'activité des insectes adultes.

Les espèces européennes à la bioécologie proche de $C$. imicola en termes de mammophilie marquée et de gîtes larvaires liés aux excréments d'animaux (C. obsoletus sensu lato ${ }^{1}, C$. pulicaris) sont qualifiées d' "espèces d'intérêt» car potentiellement vectrices de FCO du fait de ces similitudes. Leur rôle de vecteur est de plus en plus suspecté pour les raisons suivantes : (i) la présence d'indices épidémiologiques comme la circulation virale et les foyers de FCO 9 en Bulgarie en l'absence de $C$. imicola mais avec $C$. obsoletus sl (18), et la circulation virale et les foyers de FCO 2 dans les montagnes de Sicile en l'absence de $C$. imicola mais avec $C$. pulicaris (25); et (ii) la présence d'indices virologiques reposant sur des spécimens sauvages de $C$. obsoletus sl trouvés infectés en Italie continentale par les sérotypes 2 et 9 (23) et de $C$. pulicaris trouvés infectés par le sérotype 2 en zone montagneuse de Sicile (8). En outre, on remarque que ces espèces européennes connaissent une baisse brutale de leur densité adulte en plein été (juillet et août), aussi bien sur le littoral Corse que sur le littoral continental, du fait peut-être de conditions climatiques trop extrêmes. Dans le même temps, les densités adultes de $C$. imicola atteignent leur maximum. Cette espèce tropicale adaptée aux températures plus élevées et à une hygrométrie moindre pourrait occuper la niche écologique laissée vacante en été par ces espèces européennes. Le réchauffement climatique pourrait donc là aussi favoriser ce phénomène.

\section{CONCLUSION}

La surveillance entomologique sentinelle des Culicoides, vecteurs avérés et potentiels de la FCO, s'est poursuivie en France en 2004 suivant le même protocole. Dans les années à venir, elle devrait

\footnotetext{
${ }^{1}$ Complexe d'espèces jumelles comprenant, outre C. obsoletus et $C$. scoticus, espèces communes dans les élevages européens, d'autres espèces plus rares telles que $C$. montanus et $C$. dewulfi.
} 
monter en puissance afin de détecter le plus précocement possible l'introduction de $C$. imicola sur le littoral, avant que des foyers cliniques de FCO n'apparaissent. D'autre part, ce réseau sentinelle fournira des informations précieuses sur les dynamiques de populations des autres espèces européennes de Culicoides vectrices potentielles de FCO, à savoir $C$. pulicaris et $C$. obsoletus sl. La mise au point récente d'un outil moléculaire de diagnostic permet sur des groupes de Culicoides piégés de détecter très rapidement la présence ou l'absence d'au moins un spécimen du vecteur principal C. imicola (9). Cet outil permettra également aux entomologistes de s'affranchir des tâches de diagnostic dans le cadre de la surveillance pour se consacrer à des études plus spécifiques ayant trait à la bioécologie et au rôle vecteur de l'insecte, et également à la modélisation et à l'analyse du risque. En effet, l'acquisition de ces données apparaît essentielle pour contribuer à une meilleure compréhension de l'épidémiologie de la maladie, et pour élaborer et valider des modèles prédictifs basés sur l'utilisation de la télédétection (images satellitaires) et les logiciels d'analyse spatiale (systèmes d'information géographique ou SIG) afin d'identifier les zones à risque de $\mathrm{FCO}(3)$.
L'invasion de la Catalogne par C. imicola au cours de l'été 2003, le rôle vecteur potentiel d'espèces européennes de Culicoides, l'émergence récente du sérotype 4 au cours de l'automne 2003 en Sardaigne et en Corse, suivant la même dynamique spatio-temporelle que l'émergence du BTV 2 en 2000, soulignent que la FCO dans le Bassin méditerranéen reste plus que jamais d'actualité. Ceci confirme tout l'intérêt de poursuivre à l'avenir une entomosurveillance et d'amorcer des études sur la compétence vectorielle des espèces locales de Culicoides vis-à-vis des sérotypes circulant en Méditerranée.

\section{Remerciements}

Ce travail a été diligenté et financé par la Dgal/ministère français de l'Agriculture et conduit par le Cirad, département Emvt, avec la collaboration active de l'université Louis Pasteur de Strasbourg, l'EID-Méditerranée, la Ddsv Haute-Corse (2B) et la Ddsv Corse du Sud (2A). Nous tenons plus particulièrement à remercier G. Bousquet (Ddsv 2A), J. Lefebvre (DDSV 2B) et nos collègues entomologistes italiens (A.-L. Accorsi, W. Mignonne), espagnol (V. Sarto I Monteys) et suisse (A. Cagienard).

\section{BIBLIOGRAPHIE}

1. BAYLIS M., 2002. The re-emergence of bluetongue. Vet. J., 164: 5-6.

2. BAILLY-CHOUMARA H., KREMER M., 1970. Deuxième contribution à l'étude des Culicoides du Maroc (Diptera, Ceratopogonidae). Cah. Orstom, Sér. Ent. Méd. Parasitol., 8 : 383-391.

3. BAYLIS M., MELLOR P.S., WITTMANN E.J., ROGERS D.J., 2001. Prediction of areas around the Mediterranean at risk of bluetongue by modelling the distribution of its vector using satellite imaging. Vet. Rec., 149: 639-643.

4. BRAVERMAN Y., CHECHIK F., 1996. Air streams and the introduction of animal diseases borne on Culicoides (Diptera: Ceratopogonidae) into Israel. Revue sci. tech. Off. int. Epizoot., 15: 1037-1052.

5. CALISTRI P., GOFFREDO M., CAPORALE V., MEISWENKEL R., 2003. The distribution of Culicoides imicola in Italy: application and evaluation of current Mediterranean models based on climate. J. vet. Med., 50: 132-138.

6. CALLOT J., KREMER M., BAILLY-CHOUMARA H., 1970. Description de Culicoides coluzzii n. sp. (Diptera, Ceratopogonidae). Bull. Soc. Zool. Fr., 95: 709-718.

7. CAPELA R., PURSE B.V., PENA I., WITTMAN E.J., MARGARITA Y., CAPELA M., ROMAO L., MELLOR P.S., BAYLIS M., 2003. Spatial distribution of Culicoides species in Portugal in relation to the transmission of African horse sickness and bluetongue viruses. Med. Vet. Entomol., 17: 165-177.

8. CARACAPPA S., TORINA A., GUERCIO A., VITALE F., CALABRO A., PURPARI G., FERRANTELLI V., VITALE M., MELLOR P.S., 2003. Identification of a novel bluetongue virus vector species of Culicoides in Sicily. Vet. Rec., 153: 71-74.

9. CETRE-SOSSAH C., BALDET T., MATHIEU B., PERRIN A., GRILLET C., ALBINA E., 2004. Molecular detection of Culicoides spp. and Culicoides imicola, the main vector of bluetongue and African horse sickness in Africa and Europe, by ITS1rDNA PCR amplification. Vet. Res., 35: 325-337.

10. DALLAS J.F., CRUICKSHANK R.H., LINTON Y.M., NOLAN D.V., PATAKAKIS M., BRAVERMAN Y., CAPELA R., CAPELA M., PENA I., MEISWINKEL R., ORTEGA M.D., BAYLIS M., MELLOR P.S., MORDUE LUNTZ A.J., 2003. Phylogenetic status and matrilineal structure of the biting midge, Culicoides imicola, in Portugal, Rhodes and Israel. Med. vet. Entomol., 17: 379-387.

11. DELECOLLE J.C., 1985. Nouvelle contribution à l'étude systématique et iconographique des espèces du genre Culicoides (Diptera : Ceratopogonidae) du nord-est de la France. Thèse Doct., université Louis Pasteur, UER Sciences vie et terre, Strasbourg, France.
12. DELECOLLE J.C., DE LA ROCQUE S., 2002. Contribution à l'étude des Culicoides de Corse. Liste des espèces recensées en 2000/2001 et redescription du principal vecteur de la fièvre catarrhale ovine : C imicola Kieffer, 1913 (Diptera : Ceratopogonidae). Bull. Soc. Entomol. Fr., 107 : 371-379.

13. HOUGARD J.M., YAMEOGO L., PHILIPPON B., 2002. Onchocerciasis in West Africa after 2002: a challenge to take up. Parasite, 9: 105-111.

14. KREMER M., LEBERRE G., BEAUCOURNU-SAGUEZ F., 1971. Note sur les Culicoides (Diptera : Ceratopogonidae) de Corse. Description de C. corsicus n. sp. Ann. Parasitol., 46: 653-660.

15. LEFEVRE P.C., 2003. Fièvre catarrhale du mouton. In : Lefèvre P.C., Blancou J., Chermette R., éds, Principales maladies infectieuses et parasitaires du bétail. Europe et régions chaudes. Maladies bactériennes, mycoses, maladies parasitaires. Paris, France, Tec \& Doc, p. 667-686.

16. MELLOR P.S., BOORMAN J.P.T., WILKINSON P.J., MARTINEZ GOMEZ F., 1983. Potential vectors of bluetongue and African horse sickness viruses in Spain. Vet. Rec., 5: 229.

17. MELLOR P.S., BOORMAN J., BAYLIS M., 2000. Culicoides biting midges: their role as arbovirus vectors. Annu. Rev. Entomol., 45: 307340 .

18. MELLOR P.S., WITTMANN E.J., 2002. Bluetongue virus in the Mediterranean Basin 1998-2001. Vet. J., 164: 20-37.

19. MIRANDA M.A., BORRAS D., RINCON C., ALEMANY A., 2003 Presence in the Balearic Islands (Spain) of the midges Culicoides imicola and Culicoides obsoletus group. Med. Vet. Entomol., 17: 52-54.

20. RAWLINGS P., PRO M.J., PENA I., ORTEGA M.D., CAPELA R., 1997. Spatial and seasonal distribution of Culicoides imicola in Iberia in relation to the transmission of African horse sickness virus. Med. Vet Entomol., 11: 49-57.

21. RIEB J.P., 1982. Contribution à la connaissance de l'écologie et de la biologie des cératopogonidés (Diptera, Nematocera). Strasbourg, France, université Louis Pasteur, UER Vie et terre, p. 395.

22. SARTO I MONTEYS V., SAIZ-ARDANAZ M., 2003. Culicoides midges in Catalonia (Spain), with special reference to likely bluetongue virus vectors. Med. Vet. Entomol., 17: 288-293.

23. SAVINI G., GOFFREDO M., MONACO F., DE SANTIS P., MEISWINKEL R., 2003. Transmission of bluetongue virus in Italy. Vet Rec., 152: 119. 
24. SELLERS R.F., MELLOR P.S., 1993. Temperature and the persistence of virus in Culicoides spp. during adverse conditions. Revue sci. tech. Off. int. Epizoot., 12: 733-755.

25. TORINA A., CARACAPPA S., MELLOR P.S., BAYLIS M., PURSE B.V., 2004. Spatial distribution of bluetongue virus and its Culicoides vectors in Sicily. Med. Vet. Entomol., 18: 81-89.

26. WATSON R.T., CORE WRITING TEAM, Eds, 2001. Climate change: synthesis report. Geneva, Switzerland, IPCC, 184 p.

27. WIRTH W.W., MARSTON N., 1968. A method for mounting small insects on microscope slides in canada balsam. Ann. Entomol. Soc. Am., 61: $783-784$

28. WITTMAN E.J., MELLOR P.S., BAYLIS M., 2001. Using climate data to map the potential distribution of Culicoides imicola (Diptera: Ceratopogonidae) in Europe. Sci. Tech. Rev., 20: 731-740.

\section{Summary}

Baldet T., Mathieu B., Delécolle J.C., Gerbier G., Roger F. Emergence of Blue Tongue in the Mediterranean Basin and Entomological Surveillance in France

Bluetongue (BT) is an arboviral disease transmitted by biting midges (Diptera: Ceratopogonidae), which emerged in the Mediterranean in 1998 as a result of the northern spread of its main vector Culicoides imicola Kieffer 1913. In France, C. imicola was detected in the south of Corsica Island in October 2000, a few days before BT serotype 2 outbreaks. An entomological surveillance network was established in 2002 (i) in Corsica, to study the population dynamics of C. imicola and other Culicoides species associated with livestock, and (ii) on the mainland, to detect the introduction of C. imicola. Night catches were performed every three weeks on 12 sites in Corsica and monthly on 19 sites on the mainland. In 2002 in Corsica, a total of 180 night catches were performed. On average C. imicola accounted for $18 \%$ of the specimens collected and prevailed in the south of the island. Adult densities reached a peak in September. C. imicola was present on all the sites during eight months, confirming that BT main vector overwinters and is now well established on the island. Moreover, in 2003, C. imicola populations increased in the North, particularly in Balagne region. The risk of invasion in the mainland was then more significant. On the mainland, out of 233 night catches (2002-2003), two specimens of C. imicola were found in 2003 at two different sites. After extensive investigations the hypothesis of the installation of $C$. imicola was ruled out. The geographical and seasonal distribution of C. imicola and other species are discussed in relation with their bioecology and environmental factors. Entomological surveillance appears essential to better understand BT epidemiology, and to develop and validate predictive models of risk areas.

Keywords: Sheep - Culicoides imicola - Bluetongue Surveillance - Corsica - France - Mediterranean region.
29. ZIENTARA S., DE LA ROCQUE S., GOURREAU I.M., GREGORY M., DIALLO A., HENDRICKX P., LIBEAU G., SAILLEAU C., DELECOLLE J.C., 2000. La fièvre catarrhale ovine en Corse en 2000. Epidémiol. Santé Anim., 38 : 133-144.

30. ZIENTARA S., GRILLET C., DE LA ROCQUE S., GOURREAU J.M., GREGORY M., DIALLO A., HENDRICKX P., LIBEAU G., SAILLEAU C., ALBINA E., BREARD E., DELECOLLE J.C., 2001. La fièvre catarrhale ovine en Corse en 2001. Epidémiol. Santé Anim., 40: 129-137.

31. ZIENTARA S., SAILLEAU C., DAUPHIN G., ROQUIER C., REMOND E.M., LEBRETON F., HAMMOUMI S., DUBOIS E., AGIER C., MERLE G., BREARD E., 2002. Identification of bluetongue virus serotype 2 (Corsican strain) by reverse-transcriptase PCR reaction analysis of segment 2 of the genome. Vet. Rec., 150: 598-601.

Reçu le 15.07.2005, accepté le 16.01.2006

\section{Resumen}

Baldet T., Mathieu B., Delécolle J.C., Gerbier G., Roger F. Surgimiento de la fiebre catarral ovina en la Cuenca mediterránea y supervisión entomológica en Francia

La fiebre catarral ovina (FCO) es una enfermedad viral transmitida por Culicoides sp. (Diptera: Ceratopogonidae), que emerge en el Mediterráneo a partir de 1998. Este surgimiento está ligado a la propagación septentrional de su vector principal Culicoides imicola Kieffer 1913. En Francia, C. imicola fue detectado en el sur de Córcega en octubre 2000, algunos días antes de la aparición de focos de FCO serotipo 2. Una red de supervisión entomológica fue establecida en 2002: (i) en Córcega, para estudiar la dinámica de las poblaciones de $C$. imicola y de otras especies de Culicoides asociadas a los criaderos; (ii) en el continente, para detectar la introducción de $C$. imicola. Se realizaron capturas nocturnas cada tres semanas en los 12 sitios en Córcega y una vez por mes en los 19 sitios del continente. En 2002, en Córcega, se efectuó un total de 180 noches de capturas. C. imicola representa en promedio $18 \%$ de los efectivos totales capturados y predomina en el sur de la isla. Las densidades adultas alcanzan un pico en septiembre. La presencia de C. imicola en todos los sitios y durante ocho meses del año confirma que el vector principal de la FCO pasa el invierno y está instalado duraderamente en la isla. Además, en 2003, las poblaciones de C. imicola se encuentran en plena expasión en el norte, particularmente en la región Balagne. El riesgo de invasión del continente por lo tanto entonces más importante. En el continente, sobre un total de 233 noches de capturas realizadas en 2002-2003, dos especimenes de C. imicola fueron detectados en 2003 en dos sitios diferentes. Investigaciones profundas no pudieron demostrar la presencia de poblaciones locales establecidas. Las dinámicas estacionales y espaciales de $C$. imicola y de otras especies de interés se discuten con relación a su bioecología y a los factores del medio ambiente. Esta supervisión entomológica parece indispensable para una mejor comprensión de la epidemiología de la FCO, así como para elaborar y validar modelos de predicción de las zonas bajo riesgo.

Palabras clave: Ovino - Culicoides imicola - Lengua azul de los ovinos - Vigilancía - Corsega - Francia - Región mediterránea. 